\title{
Nonlinear Fractional Jaulent-Miodek and Whitham-Broer-Kaup Equations within Sumudu Transform
}

\author{
Abdon Atangana ${ }^{1}$ and Dumitru Baleanu ${ }^{2,3,4}$ \\ ${ }^{1}$ Institute for Groundwater Studies, Faculty of Natural and Agricultural Sciences, University of the Free State, \\ Bloemfontein 9300, South Africa \\ ${ }^{2}$ Department of Mathematics and Computer Sciences, Faculty of Art and Sciences, Cankaya University, Balgat, 06530 Ankara, Turkey \\ ${ }^{3}$ Department of Chemical and Materials Engineering, Faculty of Engineering, King Abdulaziz University, P.O. Box 80204, \\ Jeddah 21589, Saudi Arabia \\ ${ }^{4}$ Institute of Space Sciences, P.O. Box MG-23, R 76900, Magurele-Bucharest, Romania
}

Correspondence should be addressed to Abdon Atangana; abdonatangana@yahoo.fr

Received 15 April 2013; Accepted 28 April 2013

Academic Editor: Soheil Salahshour

Copyright (C) 2013 A. Atangana and D. Baleanu. This is an open access article distributed under the Creative Commons Attribution License, which permits unrestricted use, distribution, and reproduction in any medium, provided the original work is properly cited.

We solve the system of nonlinear fractional Jaulent-Miodek and Whitham-Broer-Kaup equations via the Sumudu transform homotopy method (STHPM). The method is easy to apply, accurate, and reliable.

\section{Introduction}

Nonlinear partial differential equations arise in various areas of physics, mathematics, and engineering [1-4]. We notice that in fluid dynamics, the nonlinear evolution equations show up in the context of shallow water waves. Some of the commonly studied equations are the Korteweg-de Vries (KdV) equation, modified KdV equation, Boussinesq equation [5], Green-Naghdi equation, Gardeners equation, and Whitham-Broer-Kaup and Jaulent-Miodek (JM) equations. Analytical solutions of these equations are usually not available. Since only limited classes of equations are solved by analytical means, numerical solution of these nonlinear partial differential equations is of practical importance. Therefore, finding new methods and techniques to deal with these type of equations is still an open problem in this area. The purpose of this paper is to find an approximated solution for the system of fractional JaulentMiodek and Whitham-Broer-Kaup equations (FWBK) via the Sumudu transform method. The fractional systems of partial differential equations under investigation here are given below.
The nonlinear FWBK equation which will be considered in this paper has the following form:

$$
\begin{gathered}
\partial_{t}^{\eta} u+u u_{x}+u_{x}+\beta u_{x x}=0, \quad 0<\eta, \mu \leq 1, \\
\partial_{t}^{\mu} v+(u v)_{x}+\alpha u_{x x x}-\beta v_{x x}=0,(x, t) \in[a, b] \times[0, T],
\end{gathered}
$$

and the nonlinear FJM equation is

$$
\begin{gathered}
\partial_{t}^{\alpha} u+u_{x x x}+\frac{3}{2} v v_{x x x}+\frac{9}{2} v_{x} v_{x x}-6 u u_{x}+6 u v v_{x}-\frac{3}{2} u_{x} v_{x}^{2} \\
=0, \\
\partial_{t}^{\mu} v+v_{x x x}-6 u_{x} v_{x}-\frac{15}{2} v_{x} v^{2}=0, \quad(x, t) \in[a, b] \times[0, T] .
\end{gathered}
$$

The system of (1) and (2) is subjected to the following initial conditions:

$$
\begin{aligned}
& u(x, 0)=f(x), \\
& v(x, 0)=g(x) .
\end{aligned}
$$


FWBK equation (1) describes the dispersive long wave in shallow water, where $u(x, t)$ is the field of horizontal velocity, $v(x, t)$ is the height which deviates from the equilibrium position of liquid, and $\alpha$ and $\beta$ are constants that represent different powers. If $\alpha=0$ and $\beta=1$, (1) reduces to the classical long-wave equations which describe the shallow water wave with diffusion [6]. If $\alpha=1$ and $\beta=0$, (1) becomes the modified Boussinesq equations [7, 8]. FJM equation (2) appears in several areas of science such as condense matter physics [9], fluid mechanics [10], plasma physics [11], and optics [12] and associates with energydependent Schrödinger potential $[13,14]$.

The paper is organized as follows. In Section 2, we introduce briefly some of the basic tools of fractional order and of the Sumudu transform method. We show the numerical results in Section 4. The conclusions can be seen in Section 5.

\section{Basic Tools}

\subsection{Properties and Definitions}

Definition 1 (see [15-24]). A real function $f(x), x>0$, is said to be in the space $C_{\mu}, \mu \in \mathbb{R}$ if there exists a real number $p>\mu$, such that $f(x)=x^{p} h(x)$, where $h(x) \in C[0, \infty)$, and it is said to be in space $C_{\mu}^{m}$ if $f^{(m)} \in C_{\mu}, m \in \mathbb{N}$.

Definition 2 (see [15-24]). The Riemann-Liouville fractional integral operator of order $\alpha \geq 0$, of a function $f \in C_{\mu}, \mu \geq-1$, is defined as

$$
\begin{gathered}
J^{\alpha} f(x)=\frac{1}{\Gamma(\alpha)} \int_{0}^{x}(x-t)^{\alpha-1} f(t) d t, \quad \alpha>0, x>0, \\
J^{0} f(x)=f(x) .
\end{gathered}
$$

Properties of the operator can be found in [15-23]; we mention only the following.

For $f \in C_{\mu}, \mu \geq-1, \alpha, \beta \geq 0$ and $\gamma>-1$

$$
\begin{gathered}
J^{\alpha} J^{\beta} f(x)=J^{\alpha+\beta} f(x), \quad J^{\alpha} J^{\beta} f(x)=J^{\beta} J^{\alpha} f(x), \\
J^{\alpha} x^{\gamma}=\frac{\Gamma(\gamma+1)}{\Gamma(\alpha+\gamma+1)} x^{\alpha+\gamma} .
\end{gathered}
$$

Definition 3. The Caputo fractional order derivative is given as follows [15-18]:

$$
\begin{aligned}
& { }_{0}^{C} D_{x}^{\alpha}(f(x)) \\
& \quad=\frac{1}{\Gamma(n-\alpha)} \int_{0}^{x}(x-t)^{n-\alpha-1} \frac{d^{n} f(t)}{d t^{n}} d t, \quad n-1 \leq \alpha \leq n .
\end{aligned}
$$

Definition 4. The Riemann-Liouville fractional order derivative is given as follows [16-24]:

$$
\begin{aligned}
D_{x}^{\alpha} & (f(x)) \\
& =\frac{1}{\Gamma(n-\alpha)} \frac{d^{n}}{d x^{n}} \int_{0}^{x}(x-t)^{n-\alpha-1} f(t) d t, \quad n-1 \leq \alpha \leq n .
\end{aligned}
$$

Definition 5. The Jumarie Fractional order derivative is given as follows [24]:

$$
\begin{aligned}
D_{x}^{\alpha}(f(x))= & \frac{1}{\Gamma(n-\alpha)} \frac{d^{n}}{d x^{n}} \\
& \times \int_{0}^{x}(x-t)^{n-\alpha-1}\{f(t)-f(0)\} d t, \\
& n-1 \leq \alpha \leq n .
\end{aligned}
$$

Lemma 6. If $m-1<\alpha \leq m, m \in \mathbb{N}$ and $f \in C_{\mu}^{m}, \mu \geq-1$, then

$$
\begin{gathered}
D^{\alpha} J^{\alpha} f(x)=f(x), \\
J^{\alpha} D_{0}^{\alpha} f(x)=f(x)-\sum_{k=0}^{m-1} f^{(k)}\left(0^{+}\right) \frac{x^{k}}{k !}, \quad x>0 .
\end{gathered}
$$

Definition 7 (partial derivatives of fractional order $[15,16,19])$. Assume now that $f(\mathbf{x})$ is a function of $n$ variables $x_{i}, i=$ $1, \ldots, n$ also of class $C$ on $D \in \mathbb{R}_{n}$. As an extension of Definition 3, we define partial derivative of order $\alpha$ for $f$ with respect to $x_{i}$ the function

$$
a \partial_{\underline{\mathbf{x}}}^{\alpha} f=\left.\frac{1}{\Gamma(m-\alpha)} \int_{a}^{x_{i}}\left(x_{i}-t\right)^{m-\alpha-1} \partial_{x_{i}}^{m} f\left(x_{j}\right)\right|_{x_{j=t}} d t
$$

where $\partial_{x_{i}}^{m}$ is the usual partial derivative of integer order $m$.

\section{Background of Sumudu Transform}

Definition 8 (see [25]). The Sumudu transform of a function $f(t)$, defined for all real numbers $t \geq 0$, is the function $F_{s}(u)$, defined by

$$
S(f(t))=F_{s}(u)=\int_{0}^{\infty} \frac{1}{u} \exp \left[-\frac{t}{u}\right] f(t) d t
$$

Theorem 9 (see [26]). Let $G(u)$ be the Sumudu transform of $f(t)$ such that

(i) $(G(1 / s) / s)$ is a meromorphic function, with singularities having $\operatorname{Re}[s] \leq \gamma$;

(ii) there exist a circular region $\Gamma$ with radius $R$ and positive constants $M$ and $K$ with $|G(1 / s) / s|<M R^{-K}$, then the function $f(t)$ is given by

$$
\begin{aligned}
S^{-1}(G(s)) & =\frac{1}{2 \pi i} \int_{\gamma-i \infty}^{\gamma+i \infty} \exp [s t] G\left(\frac{1}{s}\right) \frac{d s}{s} \\
& =\sum \text { residual }\left[\exp [s t] \frac{G(1 / s)}{s}\right] .
\end{aligned}
$$

For the proof see [26].

3.1. Basics of the Sumudu Transform Homotopy Perturbation Method. We illustrate the basic idea of this method [27-32] 
by considering a general fractional nonlinear nonhomogeneous partial differential equation with the initial condition of the following form:

$$
D_{t}^{\alpha} U(x, t)=L(U(x, t))+N(U(x, t))+f(x, t), \quad \alpha>0,
$$

subject to the initial condition

$$
\begin{gathered}
D_{0}^{k} U(x, 0)=g_{k}, \quad(k=0, \ldots, n-1), \\
D_{0}^{n} U(x, 0)=0, \quad n=[\alpha],
\end{gathered}
$$

where $D_{t}^{\alpha}$ denotes without loss of generality the Caputo fraction derivative operator, $f$ is a known function, $N$ is the general nonlinear fractional differential operator, and $L$ represents a linear fractional differential operator.

Applying the Sumudu transform on both sides of (10), we obtain

$$
\begin{aligned}
S\left[D_{t}^{\alpha} U(x, t]\right)= & S[L(U(x, t))] \\
& +S[N(U(x, t))]+S[f(x, t)] .
\end{aligned}
$$

Using the property of the Sumudu transform, we have

$$
\begin{aligned}
S[U(x, t)]= & u^{\alpha} S[L(U(x, t))]+u^{\alpha} S[N(U(x, t))] \\
& +u^{\alpha} S[f(x, t)]+g(x, t) .
\end{aligned}
$$

Now applying the Sumudu inverse on both sides of (12) we obtain

$$
\begin{aligned}
U(x, t)= & S^{-1}\left[u^{\alpha} S[L(U(x, t))]+u^{\alpha} S[N(U(x, t))]\right] \\
& +G(x, t)
\end{aligned}
$$

where $G(x, t)$ represents the term arising from the known function $f(x, t)$ and the initial conditions.

Now we apply the following HPM:

$$
U(x, t)=\sum_{n=0}^{\infty} p^{n} U_{n}(x, t) .
$$

The nonlinear term can be decomposed to

$$
N U(x, t)=\sum_{n=0}^{\infty} p^{n} \mathscr{H}_{n}(U),
$$

using the He's polynomial $\mathscr{H}_{n}(U)$ given as

$$
\begin{array}{r}
\mathscr{H}_{n}\left(U_{0}, \ldots, U_{n}\right)=\frac{1}{n !} \frac{\partial^{n}}{\partial p^{n}}\left[N\left(\sum_{j=0}^{\infty} p^{j} U_{j}(x, t)\right)\right], \\
n=0,1,2 \ldots .
\end{array}
$$

Substituting (15) and (16) gives

$$
\begin{aligned}
& \sum_{n=0}^{\infty} p^{n} U_{n}(x, t) \\
& =G(x, t)+p\left[S ^ { - 1 } \left[u^{\alpha} S\left[L\left(\sum_{n=0}^{\infty} p^{n} U_{n}(x, t)\right)\right]\right.\right. \\
& \left.\left.+u^{\alpha} S\left[N\left(\sum_{n=0}^{\infty} p^{n} U_{n}(x, t)\right)\right]\right]\right],
\end{aligned}
$$

which is the coupling of the Sumudu transform and the HPM using He's polynomials. Comparing the coefficients of like powers of $p$, the following approximations are obtained $[29,30]$ :

$$
\begin{aligned}
& p^{0}: U_{0}(x, t)=G(x, t), \\
& p^{1}: U_{1}(x, t)=S^{-1}\left[u^{\alpha} S\left[L\left(U_{0}(x, t)\right)+H_{0}(U)\right]\right], \\
& p^{2}: U_{2}(x, t)=S^{-1}\left[u^{\alpha} S\left[L\left(U_{1}(x, t)\right)+H_{1}(U)\right]\right], \\
& p^{3}: U_{3}(x, t)=S^{-1}\left[u^{\alpha} S\left[L\left(U_{2}(x, t)\right)+H_{2}(U)\right]\right], \\
& p^{n}: U_{n}(x, t)=S^{-1}\left[u^{\alpha} S\left[L\left(U_{n-1}(x, t)\right)+H_{n-1}(U)\right]\right] .
\end{aligned}
$$

Finally, we approximate the analytical solution $U(x, t)$ by truncated series:

$$
U(x, t)=\lim _{N \rightarrow \infty} \sum_{n=0}^{N} U_{n}(x, t) .
$$

The above series solutions generally converge very rapidly $[29,30]$.

\section{Applications}

In this section, we apply this method for solving the system of the fractional differential equation. We will start with (1).

4.1. Approximate Solution of (1). Following carefully the steps involved in the STHPM, after comparing the terms of the same power of $p$ and choosing the appropriate initials conditions, we arrive at the following series solutions:

$$
\begin{aligned}
u_{0}(x, t)= & G(x, t)=-\frac{c_{1}}{c_{2}}+2 c_{1} \sqrt{-\alpha-\beta^{2}} \operatorname{sech}\left(c_{1} x\right), \\
v_{0}(x, t)= & G_{1}(x, t) \\
= & -c_{1}^{2}\left(\alpha+\beta^{2}\right)+2 c_{1}^{2}\left(\alpha+\beta^{2}\right) \operatorname{sech}\left(c_{1} x\right)^{2} \\
& +2 c_{1}^{2} \beta \sqrt{-\alpha-\beta^{2}} \operatorname{sech}\left(c_{1} x\right) \tanh \left(c_{1} x\right), \\
u_{1}(x, t)= & S^{-1}\left[u^{\alpha} S\left[L\left(u_{0}(x, t)\right)+H_{0}(u)\right]\right] \\
= & \frac{c_{1}^{2} t^{\eta} \operatorname{sech}\left(c_{1} x\right)^{3}}{c_{2} \Gamma(\eta+1)} \\
& \times\left(c_{1} c_{2} \beta \sqrt{-\alpha-\beta^{2}} \cos \left(2 c_{1} x\right)\right. \\
& +4 c_{1} c_{2}\left(\alpha+\beta^{2}\right) \sinh \left(c_{1} x\right)+\sqrt{-\alpha-\beta^{2}} \\
& \times\left(-3 c_{1} c_{2} \beta\right. \\
& \left.\left.\quad+\left(c_{1}-c_{2}\right) \sinh \left(2 c_{1} x\right)\right)\right),
\end{aligned}
$$


$v_{1}(x, t)$

$$
\begin{aligned}
& =S^{-1}\left[u^{\alpha} S\left[L\left(v_{0}(x, t)\right)+H_{0}(v)\right]\right] \\
& =\frac{1}{c_{2} \Gamma(1+\mu)} \\
& \times\left(2 c _ { 1 } ^ { 2 } t ^ { \mu } \left(-2 c_{1} \operatorname{sech}(x)\left(\alpha+\beta^{2}\right)\right.\right. \\
& -2 c_{2} \beta\left(\alpha+\beta^{2}\right) \operatorname{sech}\left(c_{1} x\right)^{4} \\
& -\frac{1}{2} \sqrt{-\alpha-\beta^{2}} \operatorname{sech}\left(c_{1} x\right)^{5} \\
& \times\left(\beta-28 c_{1} c_{2} \beta^{2}+\beta\left(1+18 c_{1} c_{2} \beta\right)\right. \\
& \left.\times \cosh \left(2 c_{1} x\right)-5 c_{2} \sinh \left(2 c_{1} x\right)\right) \\
& +2 c_{2} \beta\left(\alpha+\beta^{2}\right) \operatorname{sech}\left(c_{1} x\right)^{2} \tanh \left(c_{1} x\right)^{2} \\
& +\sqrt{-\alpha-\beta^{2}} \operatorname{sech}\left(c_{1} x\right) \\
& \times\left(4 c_{1} c_{2} \operatorname{sech}(x)\left(\alpha+\beta^{2}\right)\right. \\
& +\tanh \left(c_{1} x\right)^{2} \\
& \times\left(\beta+c_{2} \tanh \left(c_{1} x\right)\right. \\
& \left.\left.\left.\left.\times\left(-1+c_{1} \beta^{2} \tanh \left(c_{1} x\right)\right)\right)\right)\right)\right), \\
& u_{2}(x, t) \\
& =S^{-1}\left[u^{\alpha} S\left[L\left(u_{1}(x, t)\right)+H_{1}(v)\right]\right]
\end{aligned}
$$$$
=\frac{1}{c_{2}^{2} \Gamma(1+2 \eta)}
$$$$
\times\left(4^{-1-\eta} c_{1}^{3} t^{2 \eta} \operatorname{sech}\left(c_{1} x\right)^{5}\right.
$$$$
\times\left(-5 \times 4^{\eta} \sqrt{-\alpha-\beta^{2}}\right.
$$$$
\left.\times\left(2 c_{1} c_{2}-c_{2}^{2}+c_{1}^{2}\left(16 \alpha+39 \beta^{2}\right)\right)\right)
$$$$
+3 \times 4^{2+\eta} c_{1}\left(c_{1}-c_{2}\right) c_{2}\left(\alpha+\beta^{2}\right) \cosh \left(c_{1} x\right)
$$$$
+4^{1+\eta} \sqrt{-\alpha-\beta^{2}}\left(-2 c_{1} c_{2}+c_{2}^{2}+c_{1}^{2}\right.
$$$$
\left.\times\left(1+c_{2}^{2}\left(12 \alpha+31 \beta^{2}\right)\right)\right)
$$$$
\times \cosh \left(2 c_{1} x\right)+4^{2+\eta} c_{1} c_{2}\left(-c_{1}+c_{2}\right)\left(\alpha+\beta^{2}\right)
$$$$
\times \cosh \left(3 c_{1} x\right)-4^{\eta} c_{1}^{2} \sqrt{-\alpha-\beta^{2}} \cosh \left(4 c_{1} x\right)
$$

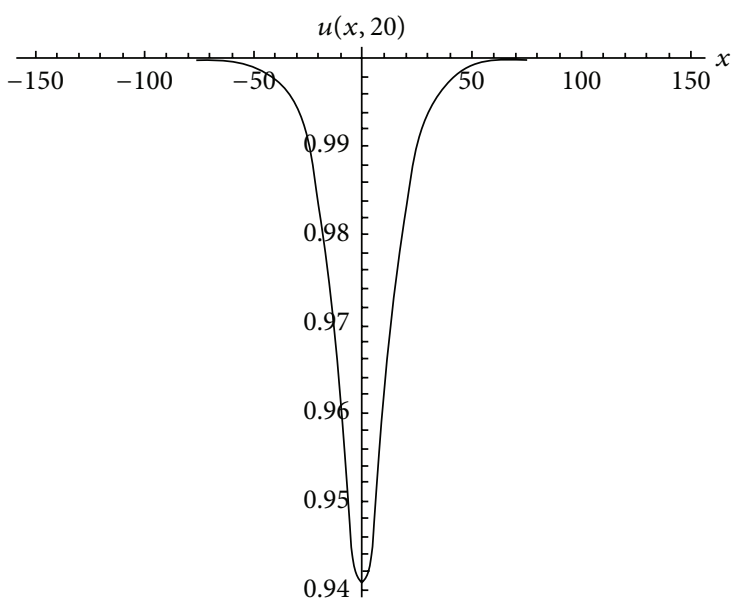

FIgURE 1: Approximate solution for FWBK equation.

$$
\begin{aligned}
& +2^{2 \eta+1} c_{1} c_{2} \sqrt{-\alpha-\beta^{2}} \cosh \left(4 c_{1} x\right) \\
& -4^{\eta} c_{2}^{2} \sqrt{-\alpha-\beta^{2}} \cosh \left(4 c_{1} x\right) \\
& -4^{\eta} c_{2}^{2} c_{1}^{2} \beta^{2} \sqrt{-\alpha-\beta^{2}} \cosh \left(4 c_{1} x\right) \\
& +11 \times 4^{1+\eta} c_{2} c_{1}^{2} \beta^{2} \sqrt{-\alpha-\beta^{2}} \sinh \left(2 c_{1} x\right) \\
& -11 \times 4^{1+\eta} c_{1} c_{2}^{2} \beta^{2} \sqrt{-\alpha-\beta^{2}} \sinh \left(2 c_{1} x\right) \\
& +2^{3+2 \eta} c_{1}^{2} c_{2}^{2} \beta\left(\alpha+\beta^{2}\right) \\
& \times\left(37 \sinh ^{2}\left(c_{1} x\right)-3 \sinh \left(3 c_{1} x\right)\right) \\
& -4^{\eta+1} c_{2} c_{1}^{2} \beta \sqrt{-\alpha-\beta^{2}} \sinh \left(4 c_{1} x\right) \\
& \left.+4^{\eta+1} c_{1} c_{2}^{2} \beta \sqrt{-\alpha-\beta^{2}} \sinh \left(4 c_{1} x\right)\right) .
\end{aligned}
$$

And so on in the same manner one can obtain the rest of the components. However, here, few terms were computed and the asymptotic solution is given by

$$
\begin{gathered}
u(x, t)=u_{0}(x, t)+u_{1}(x, t)+u_{2}(x, t)+u_{3}(x, t)+\cdots \\
v(x, t)=v_{0}(x, t)+v_{1}(x, t)+v_{2}(x, t)+v_{3}(x, t)+\cdots .
\end{gathered}
$$

Figures 1, 2, 3, and 4 show the graphical representation of the approximated solution of the system of nonlinear fractional Whitham-Broer-Kaup equation for $\eta=0.9, \mu=0.98, c_{1}=$ $c_{2}=0.1$, and $\beta=\alpha=0.1$. 


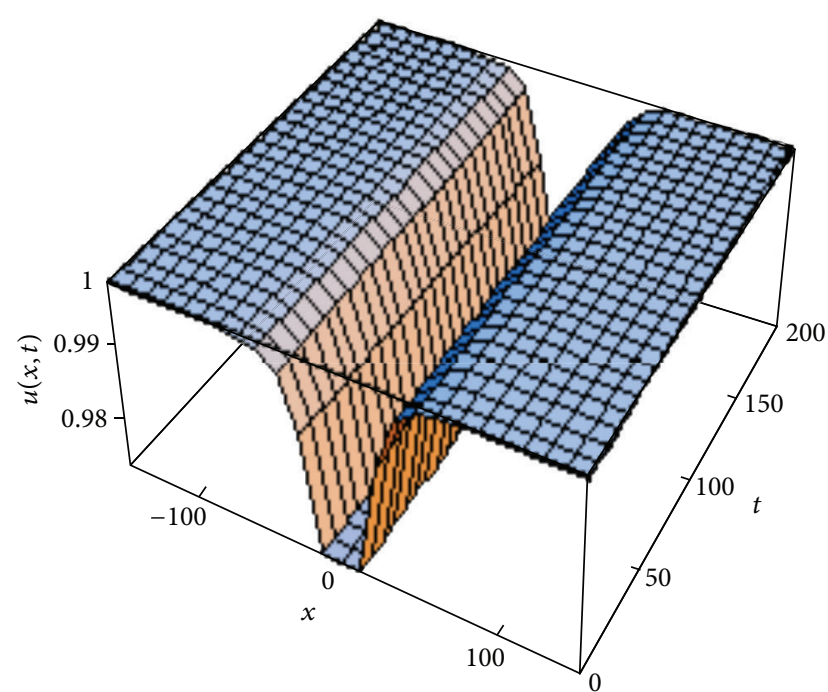

FIgURE 2: Approximate solution of FWBK equation.

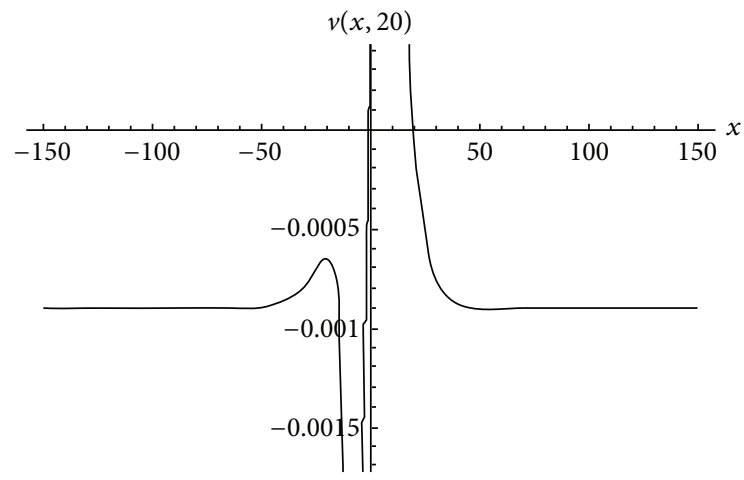

FIGURE 3: Approximate solution of FWBK equation.

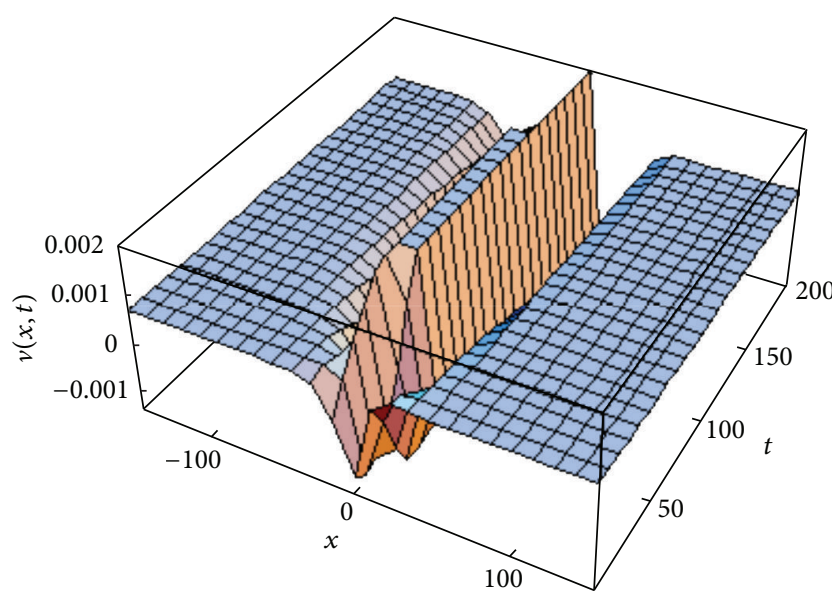

FIGURE 4: Approximate solution of FWBK equation.
4.2. Approximate Solution of (2). For (2), in the view of the Sumudu transform method, by choosing the appropriate initials conditions we are at the following series solutions:

$$
\begin{gathered}
u_{0}(x, t)=\frac{c^{2}}{8}\left(1-\operatorname{sech}\left(\frac{c x}{2}\right)^{2}\right), \\
v_{0}(x, t)=c \operatorname{sech}\left(\frac{c x}{2}\right)^{2}, \\
u_{1}(x, t)=-\frac{c^{5} t^{\eta} \operatorname{sech}(c x / 2)^{5}}{128 \Gamma(\eta+1)} \\
\quad \times\left(192 \cosh \left[\frac{c x}{2}\right]-32 \cosh \left[\frac{3 c x}{2}\right]\right. \\
\left.\quad+3 c\left(3 \sinh \left[\frac{c x}{2}\right]+\sinh \left[\frac{3 c x}{2}\right]\right)\right)
\end{gathered}
$$$$
\times \tanh \left[\frac{c x}{2}\right]
$$$$
v_{1}(x, t)=-\frac{c^{4} t^{\mu} \operatorname{sech}(c x / 2)^{3} \tanh [c x / 2]}{16 \Gamma(\mu+1)}
$$$$
\times\left(71-\cosh [c x]+6 c \tanh \left[\frac{c x}{2}\right]\right),
$$

$u_{2}(x, t)$

$$
\begin{aligned}
& =\left(\frac{4^{-10-\eta} c^{5} t^{\eta}(c x / 2)^{15}}{\Gamma(1+\mu) \Gamma(1+\eta) \Gamma(0.5+\eta) \Gamma(1+\mu+\eta)}\right. \\
& \quad \times\left(-32 c^{3} \sqrt{\pi} t^{\eta} \mu \cosh \left(\frac{c x}{2}\right)^{4} \Gamma(\mu)\right.
\end{aligned}
$$

$$
\times \Gamma(1+\eta+\mu) \Gamma(1+2 \eta+\mu)
$$

$$
\begin{aligned}
& \times\left(221184-20532 c^{2}\right) \cosh \left(\frac{c x}{2}\right) \\
& +6\left(-11008+4813 c^{2}\right) \cosh \left(\frac{3 c x}{2}\right) \\
& -69120 \cosh \left(\frac{5 c x}{2}\right)-8622 c^{2} \cosh \left(\frac{5 c x}{2}\right) \\
& +10368 \cosh \left(\frac{7 c x}{2}\right)+267 c^{2} \cosh \left(\frac{7 c x}{2}\right) \\
& -128 \cosh \left(\frac{9 c x}{2}\right)+9 c^{2} \cosh \left(\frac{9 c x}{2}\right) \\
& +61032 c \sinh \left(\frac{c x}{2}\right)-2772 c^{3} \sinh \left(\frac{c x}{2}\right) \\
& +29040 c \sinh \left(\frac{3 c x}{2}\right)+828 c^{3} \sinh \left(\frac{3 c x}{2}\right) \\
& -27312 c \sinh \left(\frac{5 c x}{2}\right)+108 c^{3} \sinh \left(\frac{5 c x}{2}\right)
\end{aligned}
$$




$$
\begin{aligned}
& +4596 c \sinh \left(\frac{7 c x}{2}\right) \\
& \left.-36 c^{3} \sinh \left(\frac{7 c x}{2}\right)-84 c \sinh \left(\frac{9 c x}{2}\right)\right) \\
& +3 \times 4^{\eta} \Gamma(0.5+\eta) \\
& \times\left(65536 \mu \cosh \left(\frac{9 c x}{2}\right)^{9} \Gamma(\mu) \Gamma(1+\eta+\mu) \Gamma\right. \\
& \times(1+2 \eta+\mu) \sinh \left(\frac{c x}{2}\right)^{2} \\
& \times(-2 c+\sinh (c x)) \\
& +1024 c^{3} t^{\eta} \eta \Gamma(\eta) \Gamma(1+\mu) \Gamma \\
& \times(1+2 \eta+\mu) \sinh \left(\frac{c x}{2}\right)^{2} \\
& \times\left(-15745 \cosh \left(\frac{c x}{2}\right)+12951 \cosh \left(\frac{3 c x}{2}\right)\right. \\
& -1175 \cosh \left(\frac{3 c x}{2}\right)+\cosh \left(\frac{7 c x}{2}\right) \\
& -6240 c \sinh \left(\frac{c x}{2}\right) \\
& +1728 c \sinh \left(\frac{3 c x}{2}\right) \\
& \left.-96 c \sinh \left(\frac{5 c x}{2}\right)\right) \\
& +2 c^{6} t^{\eta+\mu} \Gamma(1+\eta+\mu)^{2} \sinh \left(\frac{c x}{2}\right) \\
& \times\left(-235648-1154128 c^{2}+15804 c^{4}\right. \\
& -16\left(5584-7358 c^{2}+1125 c^{4}\right) \\
& \times \cosh (c x) \\
& +16\left(15904-60016 c^{2}+99 c^{4}\right) \\
& \times \cosh (2 c x) \\
& +89216 \cosh (3 c x)-296896 c^{2} \\
& \times \cosh (3 c x) \\
& +720 c^{4} \cosh (3 c x)-18816 \cosh (4 c x) \\
& +14672 c^{2} \cosh (4 c x)-108 c^{4} \\
& \times \cosh (4 c x) \\
& \left.+128 \cosh (5 c x)-32 c^{2} \cosh (5 c x)\right)
\end{aligned}
$$$$
-52680 c \sinh (c x)-391458 c^{3} \sinh (c x)
$$$$
-240 c \sinh (2 c x)+196824 c^{3} \sinh (2 c x)
$$$$
+17580 c \sinh (3 c x)-24207 c^{3} \sinh (3 c x)
$$$$
+120 c \sinh (4 c x)-156 c^{3} \sinh (4 c x)
$$$$
\left.\left.-12 c \sinh (5 c x)+3 c^{3} \sinh (4 c x)\right)\right)
$$$$
v_{2}(x, t)
$$

$$
\begin{aligned}
& =\left(2^{-17-2 \eta} c^{4} t^{\mu} \operatorname{sech}\left(\frac{c x}{2}\right)^{13}\right. \\
& \times\left(\Gamma(1+\mu)^{2} \Gamma(1+\eta) \Gamma(0.5+\mu) \Gamma\right. \\
& \times(1+\mu+\eta) \Gamma(1+3 \mu))^{-1} \\
& \times\left(3 \times 4^{4+\mu} c^{4} t^{\eta} \cosh \left(\frac{c x}{2}\right)^{4}\right. \\
& \quad \times \Gamma(1+\mu)^{2} \Gamma(1+\eta) \Gamma(0.5+\mu) \\
& \quad \times \Gamma(1+3 \mu) \sinh \left(\frac{c x}{2}\right) \\
& \times\left(896 \cosh \left(\frac{c x}{2}\right)-608 \cosh \left(\frac{3 c x}{2}\right)\right. \\
& \quad+32 \cosh \left(\frac{5 c x}{2}\right)+78 c \sinh \left(\frac{c x}{2}\right) \\
& \left.\quad+3 c \sinh \left(\frac{3 c x}{2}\right)-5 c \sinh \left(\frac{5 c x}{2}\right)\right)
\end{aligned}
$$$$
+\Gamma(\eta+1) \Gamma(1+\eta+\mu)
$$$$
\times\left(15 \times 4^{\mu} \Gamma(0.5+\mu)\right.
$$$$
\times\left(-65536 \mu \cosh \left(\frac{c x}{2}\right)^{9} \Gamma(\mu)\right.
$$$$
\left.\times \Gamma(1+3 \mu) \sinh \left(\frac{3 c x}{2}\right)\right)
$$$$
+2 c^{7} t^{2 \mu} \Gamma(1+2 \mu)
$$$$
\times\left(994 \cosh \left(\frac{c x}{2}\right)-435 \cosh \left(\frac{3 c x}{2}\right)\right.
$$$$
+\cosh \left(\frac{5 c x}{2}\right)+204 c \sinh \left(\frac{c x}{2}\right)
$$$$
\left.\left.-36 c \sinh \left(\frac{3 c x}{2}\right)-5 c \sinh \left(\frac{3 c x}{2}\right)\right)^{2}\right)
$$$$
+16 c^{3} \sqrt{\pi} t^{\mu} \mu \cosh \left(\frac{c x}{2}\right)^{4}
$$ 


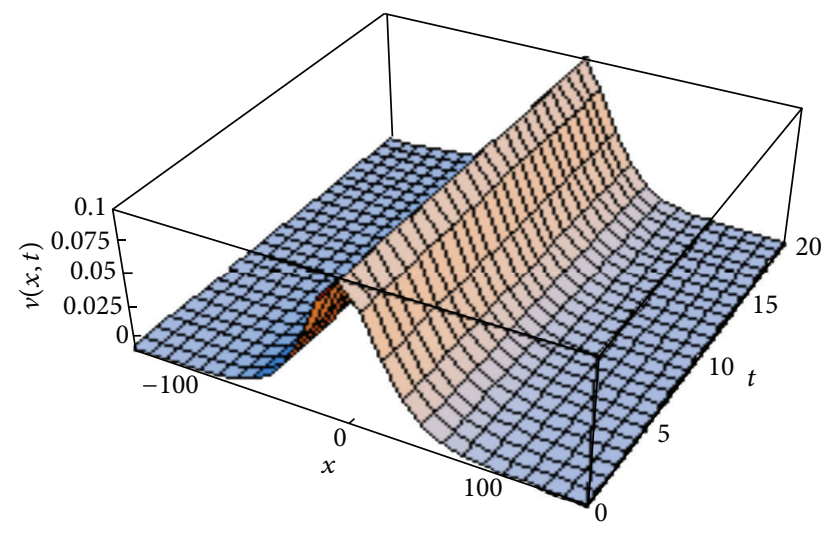

FIGURE 5: Approximate solution of FJM equation.

$$
\begin{aligned}
\times & \Gamma(\mu) \Gamma(1+3 \mu) \\
\times & \left(-79965-22032 c^{2}\right. \\
& +8\left(-2633+3240 c^{2}\right) \cosh (c x) \\
& +\left(54940-3888 c^{2}\right) \cosh (2 c x) \\
& -3960 \cosh (3 c x)+\cosh (4 c x) \\
& +130152 c \sinh (c x)-39504 c \sinh (2 c x) \\
& -216 c \sinh (3 c x)))) .
\end{aligned}
$$

And so on in the same manner one can obtain the rest of the components. However, here, few terms were computed, and the asymptotic solution of the nonlinear fractional JaulentMiodek is given by

$$
\begin{gathered}
u(x, t)=u_{0}(x, t)+u_{1}(x, t)+u_{2}(x, t)+u_{3}(x, t)+\cdots, \\
v(x, t)=v_{0}(x, t)+v_{1}(x, t)+v_{2}(x, t)+v_{3}(x, t)+\cdots
\end{gathered}
$$

Figures 5 and 6 show the graphical representation of the approximated solution of the system of nonlinear fractional Jaulent-Miodek equation for $\eta=0.98, \mu=0.48$, and $c=0.1$. Figures 5 and 6 show the approximate solution of the main problem.

\section{Conclusion}

We derived approximated solutions of nonlinear fractional Jaulent-Miodek and Whitham-Broer-Kaup equations using the relatively new analytical technique the STHPM. We presented the brief history and some properties of fractional derivative concept. It is demonstrated that STHPM is a powerful and efficient tool for the system of FPDEs. In addition, the calculations involved in STHPM are very simple and straightforward.

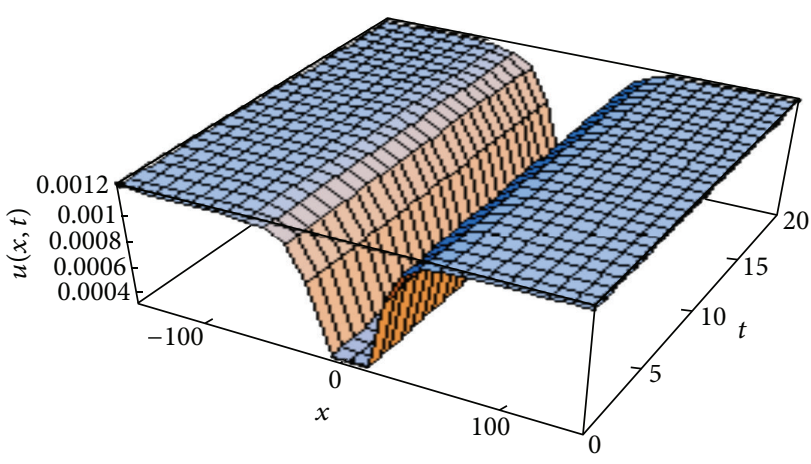

FIGURE 6: Approximate solution of FJM equation.

The STHPM is chosen to solve this nonlinear problem because of the following advantages that the method has over the existing methods. This method does not require the linearization or assumptions of weak nonlinearity. The solutions are not generated in the form of general solution as in the Adomian decomposition method (ADM) $[33,34]$. No correction functional or Lagrange multiplier is required in the case of the variational iteration method $[35,36]$. It is more realistic compared to the method of simplifying the physical problems. If the exact solution of the partial differential equation exists, the approximated solution via the method converges to the exact solution. STHPM provides us with a convenient way to control the convergence of approximation series without adapting $h$, as in the case of [37] which is a fundamental qualitative difference in the analysis between STHPM and other methods. And also there is nothing like solving a partial differential equation after comparing the terms of same power of $p$ like in the case of homotopy perturbation method (HPM) [38].

\section{References}

[1] F. Mainardi, "Fractional calculus: some basic problems in continuum and statistical mechanics," in Fractals and Fractional Calculus in Continuum Mechanics, vol. 378 of CISM Courses and Lectures, pp. 291-348, Springer, Vienna, Austria, 1997.

[2] A. Atangana and E. Alabaraoye, "Solving system of fractional partial differential equations arisen in the model of HIV infection of $\mathrm{CD}^{+}$cells and attractor one-dimensional KellerSegel equation," Advances in Difference Equations, vol. 2013, article 94, 2013.

[3] A. Atangana and A. Kılıçman, "Analytical solutions of the spacetime fractional derivative of advection dispersion equation," Mathematical Problems in Engineering, vol. 2013, Article ID 853127, 9 pages, 2013.

[4] A. Atangana and J. F. Botha, "Analytical solution of the ground water flow equation obtained via homotopy decomposition method," Journal of Earth Science \& Climatic Change, vol. 3, p. $115,2012$.

[5] N. I. Mahmudov, "Approximate controllability of fractional sobolev-type evolution equations in banach spaces," Abstract and Applied Analysis, vol. 2013, Article ID 502839, 9 pages, 2013.

[6] A. Mohebbi and Z. Asgari, "Efficient numerical algorithms for the solution of "good" Boussinesq equation in water wave 
propagation," Computer Physics Communications, vol. 182, no. 12, pp. 2464-2470, 2011.

[7] F. Xie, Z. Yan, and H. Zhang, "Explicit and exact traveling wave solutions of Whitham-Broer-Kaup shallow water equations," Physics Letters A, vol. 285, no. 1-2, pp. 76-80, 2001.

[8] B. A. Kupershmidt, "Mathematics of dispersive water waves," Communications in Mathematical Physics, vol. 99, no. 1, pp. 5173, 1985.

[9] W.-X. Ma, C.-X. Li, and J. He, "A second Wronskian formulation of the Boussinesq equation," Nonlinear Analysis. Theory, Methods \& Applications, vol. 70, no. 12, pp. 4245-4258, 2009.

[10] T. Hong, Y. Z. Wang, and Y. S. Huo, "Bogoliubov quasiparticles carried by dark solitonic excitations in non-uniform BoseEinstein condensates," Chinese Physics Letters, vol. 15, pp. 550552,1998

[11] J.-f. Zhang, "Multiple soliton solutions of the dispersive longwave equations," Chinese Physics Letters, vol. 16, no. 1, pp. 4-5, 1999.

[12] G. C. Das, J. Sarma, and C. Uberoi, "Explosion of a soliton in a multicomponent plasma," Physics of Plasmas, vol. 4, no. 6, pp. 2095-2100, 1997.

[13] S. Y. Lou, "A direct perturbation method: nonlinear Schrodinger equation with loss," Chinese Physics Letters, vol. 16, pp. 659-661, 1999.

[14] A. Atangana and A. H. Cloot, "Stability and convergence of the space fractional variable-order Schrödinger equation," Advances in Difference Equations, vol. 2013, article 80, 2013.

[15] D. Baleanu, K. Diethelm, E. Scalas, and J. J. Trujillo, Fractional Calculus Models and Numerical Methods, vol. 3 of Series on Complexity, Nonlinearity and Chaos, World Scientific, Hackensack, NJ, USA, 2012.

[16] A. A. Kilbas, H. M. Srivastava, and J. J. Trujillo, Theory and Applications of Fractional Differential Equations, vol. 204 of North-Holland Mathematics Studies, Elsevier Science B. V., Amsterdam, The Netherlands, 2006.

[17] K. B. Oldham and J. Spanier, The Fractional Calculus, Mathematics in Science and Engineering, vol. 111, Academic Press, New York, NY, USA, 1974.

[18] B. Ross, Ed., Fractional Calculus and Its Applications, vol. 457 of Lecture Notes in Mathematics, Springer, Berlin, Germany, 1975.

[19] Y. Luchko and R. Gorenflo, "The initial value problem for some fractional differential equations with the Caputo derivative," Preprint Series A08-98, Fachbreich Mathematik and Informatik, Freic Universitat Berlin, 1998.

[20] A. Atangana and A. Secer, "A note on fractional order derivatives and table of fractional derivatives of some special functions," Abstract and Applied Analysis, vol. 2013, Article ID 279681, 8 pages, 2013.

[21] I. Podlubny, Fractional Differential Equations, vol. 198 of Mathematics in Science and Engineering, Academic Press, San Diego, Calif, USA, 1999.

[22] S. G. Samko, A. A. Kilbas, and O. I. Marichev, Fractional Integrals and Derivatives: Theory and Applications, Gordon and Breach Science, Yverdon, Switzerland, 1993.

[23] K. S. Miller and B. Ross, An Introduction to the Fractional Calculus and Fractional Differential Equations, A Wiley-Interscience, John Wiley \& Sons, New York, NY, USA, 1993.

[24] G. Jumarie, "On the representation of fractional Brownian motion as an integral with respect to $(\mathrm{d} t)^{a}$," Applied Mathematics Letters, vol. 18, no. 7, pp. 739-748, 2005.
[25] G. K. Watugala, "Sumudu transform: a new integral transform to solve differential equations and control engineering problems," International Journal of Mathematical Education in Science and Technology, vol. 24, no. 1, pp. 35-43, 1993.

[26] S. Weerakoon, "Application of Sumudu transform to partial differential equations," International Journal of Mathematical Education in Science and Technology, vol. 25, no. 2, pp. 277-283, 1994.

[27] J. Singh, D. Kumar, and A. Kılıçman, "Homotopy perturbation method for fractional gas dynamics equation using Sumudu transform," Abstract and Applied Analysis, vol. 2013, Article ID 934060, 8 pages, 2013.

[28] H. Eltayeb and A. Kılıçman, "A note on the Sumudu transforms and differential equations," Applied Mathematical Sciences, vol. 4, no. 21-24, pp. 1089-1098, 2010.

[29] A. Kılıçman, H. Eltayeb, and P. R. Agarwal, "On Sumudu transform and system of differ ential equations," Abstract and Applied Analysis, vol. 2010, Article ID 598702, 11 pages, 2010.

[30] V. G. Gupta and B. Sharma, "Application of Sumudu transform in reaction-diffusion systems and nonlinear waves," Applied Mathematical Sciences, vol. 4, no. 9-12, pp. 435-446, 2010.

[31] H. Eltayeb and A. Kılıçman, "A note on the Sumudu transforms and differential equations," Applied Mathematical Sciences, vol. 4, no. 21-24, pp. 1089-1098, 2010.

[32] F. B. M. Belgacem, A. A. Karaballi, and S. L. Kalla, "Analytical investigations of the Sumudu transform and applications to integral production equations," Mathematical Problems in Engineering, no. 3-4, pp. 103-118, 2003.

[33] A. Abdon, "New cass of boundary value problems," Informations Sciences Letters, vol. 1, no. 2, pp. 67-76, 2012.

[34] G.-C. Wu, "Adomian decomposition method for non-smooth initial value problems," Mathematical and Computer Modelling, vol. 54, no. 9-10, pp. 2104-2108, 2011.

[35] G. C. Wu and D. Baleanu, "Variational iteration method for the Burgers' flow with fractional derivatives-New Lagrange multipliers," Applied Mathematical Modelling, vol. 5, pp. 10121018, 2012.

[36] G.-C. Wu and D. Baleanu, "Variational iteration method for fractional calculus-a universal approach by Laplace transform," Advances in Difference Equations, vol. 2013, article 18, 2013.

[37] H. Xu, S.-J. Liao, and X.-C. You, "Analysis of nonlinear fractional partial differential equations with the homotopy analysis method," Communications in Nonlinear Science and Numerical Simulation, vol. 14, no. 4, pp. 1152-1156, 2009.

[38] J.-H. He, "Homotopy perturbation method with an auxiliary term," Abstract and Applied Analysis, vol. 2012, Article ID 857612, 7 pages, 2012. 


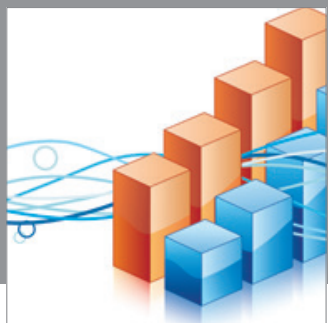

Advances in

Operations Research

mansans

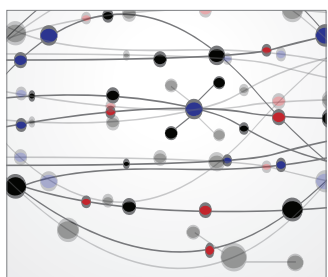

The Scientific World Journal
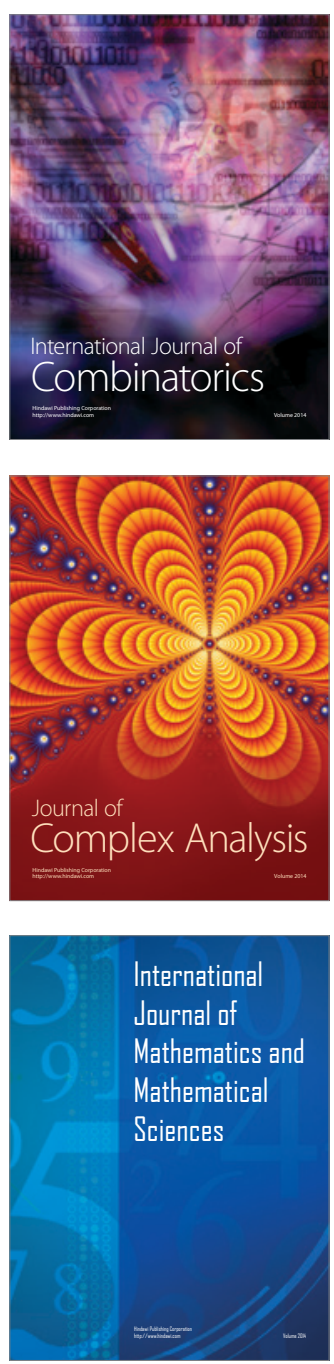
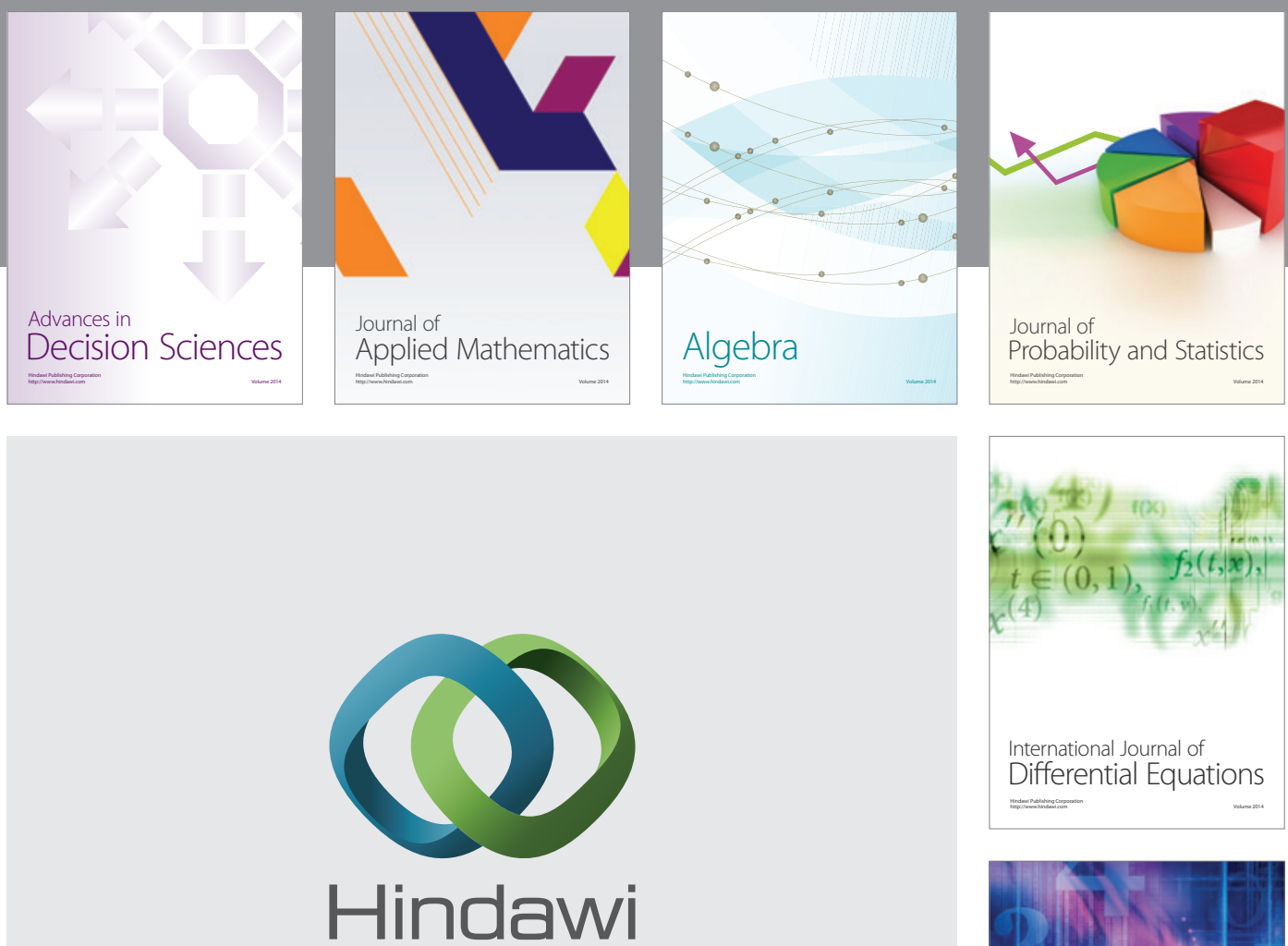

Submit your manuscripts at http://www.hindawi.com
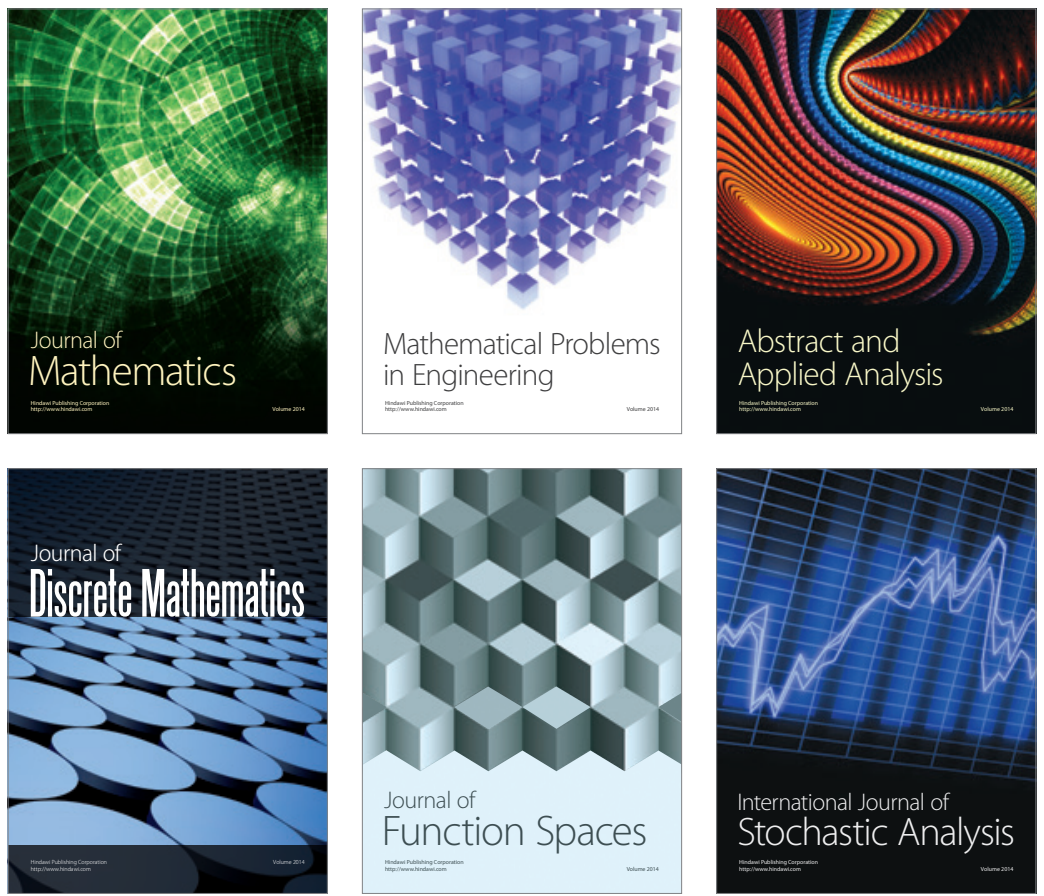

Journal of

Function Spaces

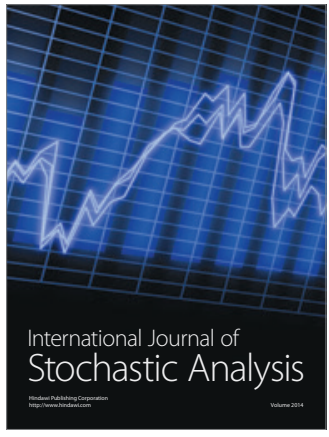

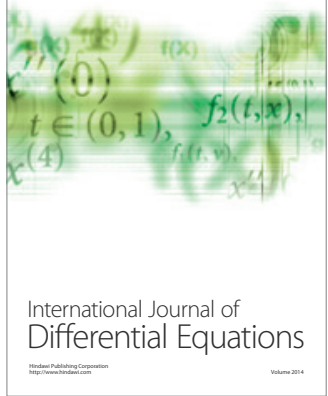
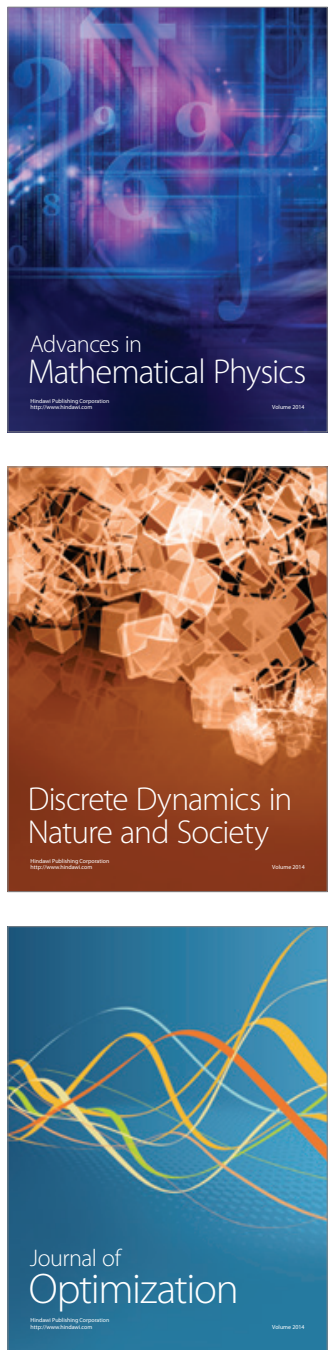